\title{
CALIDAD DE VIDA Y DIFERENCIACION RESIDENCIAL EN EL GRAN SANTA FE A COMIENZOS DEL SIGLO XXI
}

\section{QUALITY OF LIFE AND RESIDENTIAL DIFFERENTIATION IN THE SANTA FE AGGLOMERATION TO THE BEGINNING OF THE 21st CENTURY}

Prof. Dr. Javier Gómez

Profesor Asociado e Investigador Asistente. Cátedra Geografía Urbana y S.I.G.II

Departamento de Geografía. Facultad de Humanidades y Ciencias. Universidad Nacional del Litoral/Conicet

\section{RESUMEN}

Esta trabajo apunta a desentrañar la estructuración del Gran Santa Fe (AGSF) y hace hincapié en cómo las formas de habitar la ciudad por los diferentes grupos sociales configuran pero también modifican y reproducen la desigualdad urbana. Se demuestra que la forma actual del AGSF combina tres modelizaciones de ciudad latinoamericana, retomando los caracteres de la localidad y analizando su transformación espacial durante el siglo XX en el marco de las etapas económicas. El trabajo se basa en una metodología cuantitativa y utiliza como fuente principal al Censo de Población de 2001. Se apoya en los aspectos teóricos de Calidad de Vida y Ecología Factorial. Finalmente, se plantea el modelo y se describe la estructura urbana del AGSF a comienzos del S. XXI.

\section{PALABRAS CLAVES}

Diferenciación residencial; Calidad de Vida; Estructura urbana; Gran Santa Fe

\section{ABSTRACT}

This work focuses on the knowledgement of the Santa Fe Urban Agglomeration (AGSF). It emphasizes how the ways of inhabiting the city by different social groups configure but also modify and reproduce urban inequality. It shows that the actual city structure model of AGSF combines three types of latinoamerican cities, returning to the caracter of the town and analysing their spatial transformation during the 20th century in the context of the economic stages. The study is based on a cuantitative methodology and its principal source is the Population Census from 2001. It relies on the theoretical aspects of Quality of Life and Factorial Ecology. Finally, it sets the model and describes the urban structure of AGSF at the begining of 21st century.

\section{KEY WORDS}

Social-residential differentiation; Quality of life; City structure models, Santa Fe Urban Agglomeration

\section{INTRODUCCION}

Por medio de la observación directa, es posible apreciar en las ciudades latinoamericanas la coexistencia de áreas urbanas diferenciadas de acuerdo a ciertos aspectos de los grupos sociales que las habitan y de sus características físicas. Figurativamente, podríamos señalar que existen diferentes 'ciudades' dentro de una misma ciudad. La diferenciación residencial

Publicado en formato digital: Prof. Dr. Javier Gómez. CALIDAD DE VIDA Y DIFERENCIACION RESIDENCIAL EN EL GRAN SANTA FE A COMIENZOS DEL SIGLO XXI. RESÚMENES DE TESIS. Revista Geográfica Digital. IGUNNE. Facultad de Humanidades. UNNE. Año 12. No 24. Julio - Diciembre 2015. ISSN 1668-5180 Resistencia, Chaco

En: http://hum.unne.edu.ar/revistas/geoweb/default.htm 
socioeconómica 'parece inherente a la vida urbana, pero en la actualidad estaría adquiriendo mayor visibilidad' (Rodríguez y Arriagada, 2004) a partir de entender a la desigualdad como un fenómeno social que a su vez se manifiesta espacialmente. Desde esta perspectiva, se busca hacer hincapié en cómo las formas de habitar la ciudad contribuyen a modificar pero también a reproducir la desigualdad urbana.

En ese sentido, el interés de esta tesis radica en obtener conocimientos que permitan visibilizar los patrones espaciales estructurantes de la diferenciación socioresidencial en el Gran Santa Fe (AGSF), dado que hasta el momento hay escasos avances en esta materia en esa localidad y su conocimiento podría constituirse como una base para generar líneas de acción para la planificación territorial futura.

La tesis se articula en siete capítulos. El primero, se resuelve como la presentación del área de estudio, es decir la definición de la localidad, el análisis de su emplazamiento y la situación en el marco del sistema urbano argentino; además, ofrecemos un profundo análisis de la expansión, estructuración y transformación espacial del aglomerado durante el siglo XX. Los Capítulos 2 y 3, contienen los aspectos teóricos y metodológicos. Los Capítulos 4, 5 y 6 , se resuelven como el núcleo de esta investigación, dado que incluyen los aportes más sustantivos (Calidad de Vida, Ecología Factorial y Segregación Residencial Socioeconómica) y finalmente, el capítulo 7 reúne las conclusiones de la investigación y presenta el modelo de la estructura urbana del AGSF a comienzos del S. XXI.

\section{LA LOCALIDAD AGSF}

Es necesario reconocer el área seleccionada para el estudio. En este apartado se abordan, en primer lugar, los aspectos relacionados a la delimitación territorial del AGSF y se describen las características físico-naturales del sitio. En segundo lugar se establecen caracteres de su rol en el sistema urbano nacional en función de las etapas económicas ${ }^{1}$ que recorrió el país durante el S. XX. En tercer lugar, se describe la expansión urbana y la evolución demográfica del AGSF. Se finaliza con una caracterización de la diferenciación socioeconómica intraurbana actual, donde se intenta exponer que la diferenciación socioespacial del AGSF presenta una vertiente espacial que es necesario desentrañar.

El Aglomerado Gran Santa Fe es una localidad urbana que se desarrolla en el Departamento La Capital, uno de los diecinueve que constituyen la división políticoadministrativa de la provincia de Santa Fe. Se trata de una 'localidad compuesta' ${ }^{2}$, que en 2001 abarcaba una superficie aproximada de $140 \mathrm{~km}^{2}$ y contenía una población de 454.353 habitantes (INDEC). Se constituía a partir de las zonas urbanas de seis distritos ${ }^{3}$ : tres con rango municipal - Santa Fe, Santo Tomé y Recreo - y otros tantos, con carácter comunal San José del Rincón, Arroyo Leyes y Sauce Viejo ${ }^{4}$.

El aglomerado se ubica en la 'pampasia central' Frenguelli (1946), en el sector donde el río Salado se une al sistema hídrico del río Paraná. El primero atraviesa el sector oeste del

\footnotetext{
${ }^{1}$ Aldo Ferrer y Marcelo Rougier (2008) identifican cinco etapas en el proceso de desarrollo de la economía argentina.

2 INDEC (1998) El concepto de localidad: definición, estudio de caso y fundamentos teóricos metodológicos, Buenos Aires.

${ }^{3}$ En la provincia de Santa Fe, se denomina 'distrito' a la jurisdicción de un gobierno local y en la actualidad son 363.

${ }^{4}$ Según las Leyes Orgánicas de Municipios № 2756 y de Comunas № 2439 de la Provincia de Santa $\mathrm{Fe}$, los 363 distritos -jurisdicciones de gobiernos locales- que actualmente componen la Provincia, jurídicamente se diferencian en municipios y comunas según alcancen o no un umbral demográfico fijado en 10000 habitantes. En este sentido, los municipios se encuentran regidos por un Intendente Municipal y un Concejo Municipal, y las comunas por una Comisión Comunal.
}

Publicado en formato digital: Prof. Dr. Javier Gómez. CALIDAD DE VIDA Y DIFERENCIACION RESIDENCIAL EN EL GRAN SANTA FE A COMIENZOS DEL SIGLO XXI. RESÚMENES DE TESIS. Revista Geográfica Digital. IGUNNE. Facultad de Humanidades. UNNE. Año 12. N 24. Julio - Diciembre 2015. ISSN 1668-5180 Resistencia, Chaco

En: http://hum.unne.edu.ar/revistas/geoweb/default.htm 
aglomerado -entre los municipios de Santa Fe y Santo Tomé-. El segundo, marca su presencia en el sector oriental. El sitio se caracteriza por un relieve plano de exigua pendiente y escasa altitud, lo que le otorga marcada vulnerabilidad hídrica.

Hacia 2001, el Gran Santa Fe reunía al 15,3 \% de los habitantes de la provincia, mientras en 1895 a sólo el 7.7 \%. Esta duplicación de su representatividad se une además a cambios acaecidos en la estructura urbana a través del tiempo en el contexto de las 'etapas' económicas argentinas. La ciudad de Santa Fe tiene un origen colonial y mantuvo esa fisonomía durante tres siglos hasta que se vio alterada a partir del advenimiento de la etapa 'economía primaria exportadora', cuando se convirtió en un importante puerto (1885) y en epicentro económico y de comunicaciones producto del desarrollo ferroviario. La inmigración y el movimiento económico fueron fundamentales en su transformación y crecimiento espacial. A partir de los cambios económicos operados luego de 1930 aparecieron las primeras villas miseria en el oeste de la ciudad de Santa Fe y la escala urbana alcanzada impulsó la construcción de avenidas urbanas, que comenzaron a vertebrar la expansión. Desde mediados de la década de 1960, cobró significación la radicación de talleres e industrias metalmecánicas en el sector ubicado entre Santo Tomé y Sauce Viejo, dando lugar a la erección de numerosos barrios obreros que aceleraron la expansión horizontal de localidades suburbanas.

Hacia fines de la década de 1970, las políticas neoliberales dispararon un proceso de 'desindustrialización' y a inicios de los '80 las tasas de desempleo saltaron del 4,8\% al $10,7 \%$ (INDEC e IPEC). Hacia esta época, la expansión horizontal continuó hacia el sector norte, mientras que en el sector oriental fue ganando entidad el desarrollo de viviendas de fin de semana.

La reafirmación del proyecto neoliberal a partir de 1990 trajo aparejada una nueva contracción del sector industrial. Cobró auge el desarrollo de un nuevo frente de expansión de Santa Fe sobre la zona norte del municipio de Santo Tomé, protagonizado por emprendimientos inmobiliarios privados cuya planificación se orienta a sectores sociales medio-altos, vertebrado por la Autopista Brigadier López. Ya en la primera década del S. XXI, se aceleró en el macrocentro de Santa Fe el crecimiento vertical producto del incremento en la construcción de edificaciones con destino de uso 'multiviviendas'.

En los últimos cincuenta años, las mayores tasas de crecimiento demográfico se han centrado fundamentalmente en las localidades que rodean la ciudad central, es decir en las 'coronas', lo que indudablemente incidió en que éstas aumenten su 'peso' absoluto y relativo en el conjunto del AGSF. Este desbalance demográfico se complementa con un proceso de redistribución de la población entre las seis localidades.

El análisis de indicadores demográficos, socioeconómicos y de infraestructura y servicios juegan un importante papel en los diferentes patrones de localización de la población. Sin embargo, es posible pensar en la existencia de algunos patrones de distribución comunes. Por una parte, un grupo de variables presenta una distribución geográfica con un gradiente centro periferia. De modo complementario, y atravesando ambas categorías, otros indicadores tienden a desdibujar esa polarización. En suma, esta dualidad, junto a sus mixturas, torna evidente cada vez más las contradicciones urbanas lo cual, parafraseando a Roccatagliata (2008), hace que el estudio de estas temáticas adquiera 'vital importancia'.

\section{ASPECTOS CONCEPTUALES}

Se parte de la idea de que la intensidad de las condiciones sociales en ciertos sectores del territorio contrasta con la debilidad en otros. Según señala Ortega Valcárcel (2000) 'son los procesos de diferenciación los que destacan como los más relevantes en la construcción del mundo actual y como los que dominan a escala planetaria, estatal y local'. Al respecto, el

Publicado en formato digital: Prof. Dr. Javier Gómez. CALIDAD DE VIDA Y DIFERENCIACION RESIDENCIAL EN EL GRAN SANTA FE A COMIENZOS DEL SIGLO XXI. RESÚMENES DE TESIS. Revista Geográfica Digital. IGUNNE. Facultad de Humanidades. UNNE. Año 12. N 24. Julio - Diciembre 2015. ISSN 1668-5180 Resistencia, Chaco

En: http://hum.unne.edu.ar/revistas/geoweb/default.htm 
autor afirma que los procesos de diferenciación responden a la acción de los agentes sociales y se traduce en áreas diferenciadas por el grado de desarrollo, por la mayor o menor intensidad o acumulación...' de algún aspecto relevante.

La geografía urbana brinda una serie de perspectivas para el estudio de la diferenciación socioespacial en los espacios urbanos. Podríamos suponer que las perspectivas teóricas y metodológicas se desarrollan dentro de un marco evolutivo en el cual las más nuevas desplazan a las anteriores. Sin embargo, preferimos entender que las perspectivas que fueron surgiendo desde mediados del S. XX son resignificadas con el transcurso del tiempo, lo que enriquece el campo de estudios de la geografía urbana. Por ello, en nuestra visión consideramos que en la actualidad conviven en el seno de la geografía urbana un 'grupo' de enfoques y creemos que 'tomar' porciones de una forma más o menos ecléctica de cada una de ellas, conduciría a plantear diferentes miradas del problema, ampliando la visión de la diferenciación intraurbana.

La estructura contemporánea de los usos del suelo urbano, en Argentina, ha podido ser definida 'a través de tres patrones espaciales diferentes superpuestos en forma parcial', de lo que resultaría (Buzai, 2003b):

- 'una estructura antigua de anillos concéntricos: la estructura espacial urbana surge de la ciudad colonial, al respecto se verifica el CBD central, una anillo de usos mixtos dentro del cual se encuentran residencias, comercios, industrias, y sobre el borde de la ciudad, zonas residenciales bajas que se distribuyen en forma de manchones.

- Una estructura moderna sectorial generada principalmente a partir de la década de 1930 y corresponde al corrimiento por ejes de las zonas residenciales en las ciudades de mayor crecimiento. Por una lado, la suburbanización de los grupos sociales de alto nivel económico siguiendo la dirección de las áreas comerciales y por el otro, líneas industriales que concentran población de bajos recursos, gran parte llegada de procesos migratorios.

- Una estructura celular en la periferia: Bähr y Mertins (1982) consideran que esta estructura está principalmente formada por barrios informales (ilegales y semilegales), proyectos gubernamentales de vivienda destinados a poblaciones de recursos medios y bajos y una expansión de grupos altos en ubicaciones separada del sector de crecimiento. Mertins (1995) incluye en el esquema de barrios residenciales cerrados para poblaciones de alto nivel económico'.

La heterogeneidad social de los espacios urbanos presentaría correlatos espaciales específicos. Este razonamiento refiere a que la diferenciación residencial relacionada con esa heterogeneidad no es aleatoria, sino que en virtud de la literatura disponible y de las modelizaciones aplicables, está relacionada fundamentalmente con cuestiones relativas a las características socioeconómicas y a las condiciones de vida de la población, entre otras. Y en ese sentido, la primera hipótesis que sostenemos expresa que 'La configuración espacial que resulta de la disposición de los diversos grupos socioeconómicos en el AGSF puede asimilarse a una combinación de los modelos de estructura interna propuestos por Ford (1996), Mertins (1995) y Janoschka (2002) para el mundo urbano latinoamericano'.

Con relación a lo anterior, la segunda hipótesis destacada de nuestro estudio sostiene que 'la diferenciación residencial del AGSF puede ser explicada, a partir de dos dimensiones relevantes: el 'estatus social' y el 'estatus familiar'.

En tercer lugar, creemos que al menos dos aspectos se destacan en el análisis de la segregación residencial socioeconómica: los criterios para la determinación de los grupos socioeconómicos y la escala que se tome como referencia para el análisis. Creemos que es conveniente cruzar ambos aspectos para establecer un diagnóstico acerca del nivel de segregación. 'En el AGSF los grupos socioeconómicos exhiben diferentes grados de segregación, siendo mayor en el agregado asociado al nivel social bajo, producto de la

Publicado en formato digital: Prof. Dr. Javier Gómez. CALIDAD DE VIDA Y DIFERENCIACION RESIDENCIAL EN EL GRAN SANTA FE A COMIENZOS DEL SIGLO XXI. RESÚMENES DE TESIS. Revista Geográfica Digital. IGUNNE. Facultad de Humanidades. UNNE. Año 12. № 24. Julio - Diciembre 2015. ISSN 1668-5180 Resistencia, Chaco

En: http://hum.unne.edu.ar/revistas/geoweb/default.htm 
importante homogeneidad social de las áreas que ocupan. A su vez, por disponerse esas zonas de modo contiguo, conducirían a una segregación 'por localización de grupo' de ese colectivo social'.

Dado que al menos pueden distinguirse dos dimensiones de la calidad de vida, las 'públicas' y las 'privadas', donde en general, la primera dimensión está referida a aspectos macro, vinculados con cuestiones ambientales y de accesibilidad, mientras que la segunda depende de indicadores micro, asociados con el nivel de ingresos, la composición del grupo familiar o el nivel de instrucción. Para un análisis con escala detallada (por ejemplo una ciudad, según radios censales) es posible considerar el peso de ambas dimensiones (pública y privada) para la determinación de niveles de bienestar según sectores y grupos sociales. Así, en una ciudad, los sectores de bajos ingresos en general estarán perjudicados 'privadamente' porque sus medios no les permiten tener una vivienda confortable, alcanzar cierto nivel de instrucción o alimentarse adecuadamente pero, adicionalmente, los sectores de bajos ingresos localizados en la periferia urbana resultan perjudicados 'públicamente' porque su accesibilidad a ciertos bienes o servicios es menor que la de aquellos que residen en el centro de la ciudad. En este marco entonces, planteamos como cuarta hipótesis que ambas dimensiones intervienen en la diferenciación socio-espacial urbana, y en general, lo hacen en forma conjunta'.

\section{METODOS}

La estructura general que aborda este estudio se basa en una metodología cuantitativa, apelando primordialmente a fuentes de datos censales, y la misma se integra de tres aproximaciones: primero, el abordaje de la diferenciación por medio del enfoque de la calidad de vida; segundo, desde el enfoque de la ecología factorial y tercero, desde el examen de la segregación residencial. El censo de población constituye una base de información de uso generalizado para el estudio de estas problemáticas, destacándose además como una fuente exhaustiva de datos. Aun así es necesario tomar con precaución esos datos porque, como comúnmente se advierte, 'ni son todos los que están, ni están todos los que son'. El Censo Nacional de Población, Hogares y Vivienda es llevado a cabo por el Instituto Nacional de Estadísticas y Censos de la República Argentina (INDEC) y en términos generales, sus resultados son insumos básicos para formular políticas y administrar programas en diversas esferas. Su información permite conocer la magnitud de la población según condiciones de vida diferenciales, obtener muestras representativas para analizar la situación demográfica, social y económica de la población durante los años siguientes a la realización del Censo (Documentos Base REDATAM ${ }^{5}$ INDEC, 2003).

Es la única fuente de datos del país que provee información universal de población, hogares y viviendas hasta los niveles geográficos más pequeños. En efecto, esa fuente de información brinda los datos desagregados a diferentes escalas. Además, es la única fuente que ofrece datos por radios censales, que es la unidad de superficie más reducida para la cual la información está disponible. Esta unidad, que raramente sobrepasa los 1000 habitantes y la cantidad de habitantes promedio en el AGSF es de 1147 habitantes.

Es necesario plantear que la elección y utilización de la información censal actúa a su vez como corset y 'limita' en parte las posibilidades para la selección de las variables del estudio y la escala de análisis. El último censo del país con el que se pudo contar para realizar este

\footnotetext{
5 'REDATAM' es acrónimo de REcuperación de DATos para Áreas pequeñas por Microcomputador. La primera generación de Redatam, para DOS, se lanzó en 1987. Todas las generaciones de Redatam han sido desarrolladas por el Centro Latinoamericano y Caribeño de Demografía (CELADE), División de Población de la CEPAL.
}

Publicado en formato digital: Prof. Dr. Javier Gómez. CALIDAD DE VIDA Y DIFERENCIACION RESIDENCIAL EN EL GRAN SANTA FE A COMIENZOS DEL SIGLO XXI. RESÚMENES DE TESIS. Revista Geográfica Digital. IGUNNE. Facultad de Humanidades. UNNE. Año 12. N 24. Julio - Diciembre 2015. ISSN 1668-5180 Resistencia, Chaco

En: http://hum.unne.edu.ar/revistas/geoweb/default.htm 
trabajo, fue levantado en noviembre de 2001, en un momento coyuntural del país, el cual se debatía, por aquellos días, en una grave crisis económica, que tuvo graves implicancias políticas y sociales.

En el ámbito científico, los datos censales contribuyen a fundamentar líneas de investigación sobre la evolución de la población por sexo y edad, la estructura económico-social de la Argentina, el crecimiento poblacional pasado y reciente, cambios en la distribución de la población urbana y rural, la evolución de la fecundidad, las características de las migraciones, etcétera. La base de datos REDATAM utilizada en nuestro estudio es el resultado de un convenio de cooperación entre el INDEC y el CELADE a los fines de conformar una base de microdatos del Censo Nacional de Población, Hogares y Viviendas 2001 adaptada para usuarios que dispongan de microcomputadoras resguardando la confidencialidad de los datos individuales.

Debemos hacer la salvedad que en la Argentina los relevamientos censales no suelen recavar información sobre los ingresos de la población, no obstante, ese aspecto suele inferirse de las calificaciones laborales o los años de estudios de los jefes de hogar, denominándolas variables 'proxy' del ingreso (Kaztman, 1999).

El abordaje cuantitativo de la diferenciación socioespacial a escala urbana, requiere disponer de datos universales en su mayor desagregación posible. Como señalamos previamente, la base de información relevada por los Censos se encuentra disponible en el interior urbano a escala de fracciones y radios censales. En este marco, se torna indispensable evaluar la utilidad de ambas opciones. Las 27 fracciones censales con que cuenta el AGSF delimitan áreas urbanas notablemente extensas y con proporciones importantes de población en su interior. Los radios, en cambio, son notablemente de menor extensión, y de modo consiguiente, agrupan menor población en su interior. Para el análisis de la diferenciación socioespacial, es fundamental contar con unidades espaciales con el menor contraste posible en su interior, para procurar reducir el denominado PUEM ${ }^{6}$ (Problema de la Unidad Espacial Modificable). Este problema se produce cuando la unidad estadística no presenta homogeneidad interna, y por lo tanto el valor asociado a ella es un promedio de la realidad imperante en su interior, ocultando situaciones de heterogeneidad interna a la unidad estadística.

La desagregación de la información según los 396 radios censales incluidos en el AGSF, unidades de notable menor tamaño, posibilita contar con mayor detalle y homogeneidad de la información socioeconómica, restando probabilidades a que se suscite el PUEM. A su vez, dado que los radios censales se constituyen como el recorte territorial georeferenciado de mayor desagregación disponible, se consideran apropiados en términos operativos para avanzar con dos de los objetivos centrales de este estudio: la cuantificación y representación cartográfica de la diferenciación.

Dadas las limitaciones en la obtención de información censal a niveles geográficos más bajos, en la literatura se ha utilizado de modo generalizado a los radios censales dado que son la unidad menor de información censal que se encuentra disponible en lo que respecta a las características demográficas, económicas y sociales con aceptable nivel de homogeneidad.

\section{CALIDAD DE VIDA EN EL AGSF}

En este apartado se demuestra de qué manera esta propuesta teórico-metodológica presenta relevancia para evidenciar la diferenciación socio-residencial. Primeramente se presentan las variables e indicadores relacionados a las dimensiones 'privadas' de los

\footnotetext{
${ }^{6}$ A este concepto se refieren ampliamente Buzai (2003 y 2006), y Velázquez (2001 y 2005).
} 
hogares que seleccionamos para este estudio son: a) salud, b) educación y c) vivienda (Cuadro 1).

Cuadro 1. AGSF. Dimensiones 'Privadas' de la Calidad de Vida (ID Privadas)

\begin{tabular}{lll}
\hline Variable & Indicador & $\begin{array}{l}\text { Nombre } \\
\text { Abrev. }\end{array}$ \\
\hline Salud & Proporción de población con cobertura de Obra Social & TENOSOC \\
\hline \multirow{2}{*}{ Educación } & $\begin{array}{l}\text { Proporción de población que ya no asiste a establecimiento } \\
\text { educativo, con Nivel Educativo Primario Incompleto. }\end{array}$ & SINPRIM \\
& $\begin{array}{l}\text { Proporción de población con Nivel Educativo Universitario } \\
\text { Completo. }\end{array}$ & UNIV \\
\hline \multirow{2}{*}{ Vivienda } & $\begin{array}{l}\text { Proporción de población que carece de inodoro. } \\
\text { Proporción de población hacinada -Personas viviendo en en } \\
\text { hogares con más de 2 personas por cuarto-. }\end{array}$ & HACIN \\
\hline
\end{tabular}

Fuente: Elaboración personal sobre la base de datos proporcionados por el Censo Nacional de Población, Hogares y Viviendas (INDEC). Año 2001.

En tanto, las variables 'públicas', se presentan en el Cuadro 2.

Cuadro 2. AGSF. Dimensiones 'Públicas' de la Calidad de Vida (ID Públicas)

\begin{tabular}{lll}
\hline Variable & Indicador & $\begin{array}{l}\text { Nombre } \\
\text { Abrev. }\end{array}$ \\
\hline & Proporción de población con Descarga a red (Cloacas) & DESRED \\
$\begin{array}{l}\text { Servicios } \\
\text { Públicos }\end{array}$ & Proporción de población con Gas de red. & GASRED \\
\hline
\end{tabular}

Fuente: Elaboración personal sobre la base de datos proporcionados por el Censo Nacional de Población, Hogares y Viviendas (INDEC). Año 2001.

A partir de establecer las cuatro categorías de calidad de vida (más favorable, favorable, desfavorable y más desfavorable) fue posible dar evidencias de la configuración espacial de la diferenciación espacial socioeconómica existente en el AGSF.

Podemos señalar que la distribución espacial de la población de mayor calidad de vida se sitúa en el área central y en una corona de barrios pericentrales, en tanto la población de menor calidad de vida se ubica en la periferia del aglomerado. La población perteneciente a las categorías intermedias, se localiza fundamentalmente de modo intermedio entre las áreas previamente explicitadas. En tanto, aparecen algunas excepciones a esa generalidad, producto de situaciones excepcionales como el crecimiento de los barrios cerrados de clases altas en la periferia y la presencia de sectores socialmente desfavorecidos en áreas próximas al macrocentro de la ciudad principal del AGSF.

Publicado en formato digital: Prof. Dr. Javier Gómez. CALIDAD DE VIDA Y DIFERENCIACION RESIDENCIAL EN EL GRAN SANTA FE A COMIENZOS DEL SIGLO XXI. RESÚMENES DE TESIS. Revista Geográfica Digital. IGUNNE. Facultad de Humanidades. UNNE. Año 12. NN 24. Julio - Diciembre 2015. ISSN 1668-5180 Resistencia, Chaco

En: http://hum.unne.edu.ar/revistas/geoweb/default.htm 
Según lo evidenciado, el área de la categoría 'más favorable' de calidad de vida presenta principalmente una implantación 'central' en el AGSF. Desde esta área se observa una expansión hacia el noreste de la ciudad, donde alcanza la periferia urbana. Santo Tomé presenta también un área de esta categoría, aunque de notable menor extensión y a la cual deben agregarse otros casos de localización periférica de esta categoría: el barrio de countries en el norte de la ciudad, sobre la traza de la autopista Brigadier López, que asegura la comunicación de esta zona con la ciudad de Santa Fe, y la zona de la Unidad Militar.

El área de calidad de vida 'favorable', se muestra visiblemente adosada a modo de 'anillo' del área de mayor calidad de vida. Como se había explicitado en la Introducción esta área se corresponde principalmente con barrios surgidos en los últimos setenta años, y que han alcanzado cierto grado de madurez demográfica, a la vez que han logrado abastecerse de servicios públicos básicos, en un marco de edificación consolidada. Ello contribuye a configura a esta área como urbanísticamente 'madura'.

Fuera de estas áreas, en amplios sectores periféricos de Santa Fe y Santo Tomé, y en general, del AGSF, se observan los sectores urbanos ocupados por las categorías 'desfavorables' de calidad de vida. Si bien es clara su localización periférica, aparece configurada sobre una trama de importantes vías de circulación urbanas, que marcarían cierta influencia positiva frente a un contexto sociourbano notablemente deteriorado. Podemos citar el caso de las Avenidas Blas Parera, Peñaloza, Facundo Zuviría al final, Estanislao Zeballos y Gorriti. El área de la categoría 'más desfavorable' está asociada fundamentalmente a sectores urbanos de marcada vulnerabilidad hídrica. Tanto por la vecindad con el río Salado, en el oeste de la ciudad de Santa Fe, como por la del Paraná, en el este del AGSF.

A partir del planteo del análisis de la calidad de vida, sobre la base de las dimensiones públicas (relacionadas con las condiciones externas a los hogares) y privadas (más vinculadas con las características internas a los hogares), se ha encontrado que las configuraciones espaciales de ambas dimensiones tienden, de modo importante, a ser coincidentes, tanto en las áreas centrales como en las periféricas. Sin embargo, en las áreas centrales ambas dimensiones coinciden por el alto logro que en ellas que alcanza la población. En tanto, en las zonas periféricas, lo hacen por el menor logro que alcanza la población residente, en ellas.

Ahora bien, lo anterior no es una generalidad. No siempre los sectores urbanos donde residen los grupos sociales con dimensiones privadas satisfechas, cuentan con la presencia de los servicios públicos analizados. La coincidencia entre ambas dimensiones es más general, en los sectores de mayor antigüedad y consolidación urbanística, destacándose las áreas centrales de las principales ciudades. En cambio, aquellos sectores periféricos, donde la población posee las dimensiones 'privadas' logradas, evidencian déficits en la provisión de servicios públicos. Consideramos que las dimensiones 'privadas' y 'públicas' presentan un patrón de distribución similar, que coincide en lo global. Sin embargo, aparecen algunas excepciones. Quizás la más notable, es la protagonizada por los radios censales donde se encuentran radicados los barrios cerrados.

En suma, se denota que la diferenciación de la población en términos sociales y económicos presenta una variable espacial. La población se encuentra segmentada espacialmente en función de su nivel educativo, sus condiciones habitacionales, sanitarias y de servicios públicos.

En relación con otros estudios de diferenciación social intraurbana realizados en Argentina con el objetivo de explicar la estructura urbana en ciudades argentinas de tamaño intermedio (cfr. Milanesio de Reñé, 1994; Buzai, 2003b; Natera, 2005 y Natera y Gómez, 2007), podemos afirmar que las características de la diferenciación observadas en el AGSF

Publicado en formato digital: Prof. Dr. Javier Gómez. CALIDAD DE VIDA Y DIFERENCIACION RESIDENCIAL EN EL GRAN SANTA FE A COMIENZOS DEL SIGLO XXI. RESÚMENES DE TESIS. Revista Geográfica Digital. IGUNNE. Facultad de Humanidades. UNNE. Año 12. NN 24. Julio - Diciembre 2015. ISSN 1668-5180 Resistencia, Chaco

En: http://hum.unne.edu.ar/revistas/geoweb/default.htm 
al menos, grosso modo, se asemeja a los modelos para las ciudades argentinas y latinoamericanas de tamaño intermedio.

Sin bien se ha señalado algunos aspectos explicativos de tal configuración espacial, creemos que es necesario en lo subsiguiente indagar especialmente acerca de las causas o factores subyacentes de estas disparidades espaciales, cuestión que abordaremos a continuación.

\section{FACTORES DE DIFERENCIACIÓN RESIDENCIAL}

En este apartado la argumentación central se estructura sobre el objetivo de identificar las dimensiones que subyacen en la diferenciación socio residencial de la población del Gran Santa Fe, atendiendo no sólo a la identificación de las mismas, sino también a su análisis espacial, buscando comprender la formación de áreas residenciales de características diferenciadas. La selección de variables puede dividirse en siete grupos: primero, las características de la estructura de la población; segundo, aquellas variables que refieren a la relación entre población y viviendas; tercero, las características de los niveles educativos de la población; cuarto, indicadores que refieren al origen de la población; el quinto, las características laborales de la población; el sexto, una serie de indicadores que atañen a la pobreza estructural de la población, y el séptimo, servicios básicos de la población.

Las 22 variables empleadas en este estudio son las siguientes:

-Porcentaje de población menor de 15 años.

-Porcentaje de población de 65 y más años.

-Porcentaje de población que reside en viviendas con más de dos personas por cuarto.

-Porcentaje de población que no asiste a establecimiento escolar pero asistió, con máximo nivel de instrucción primaria incompleta.

-Porcentaje de población que no asiste a establecimiento educativo pero asistió, con máximo nivel de instrucción universitario completa.

-Porcentaje de población que asiste a centro escolar privado sobre el total de la población que asiste a un centro escolar.

-Porcentaje de población nacida en otra provincia argentina.

-Porcentaje de población que hace cinco años vivía en otra provincia argentina.

-Porcentaje de población que hace cinco años vivía en otra localidad de esta provincia (Santa Fe).

-Porcentaje de población en casas A y departamentos.

-Porcentaje de población en hogares afectados por NBI subsistencia.

-Porcentaje de población que carece de inodoro.

-Porcentaje de población sin agua corriente.

-Porcentaje de ocupados con calificación laboral no calificada.

-Porcentaje de ocupados con calificación laboral 'profesionales'.

-Porcentaje de ocupados con condición laboral 'patrones'.

-Porcentaje de población que reside en hogares con ambos jefes desocupados.

-Porcentaje de población que reside en viviendas con piso de tierra.

-Porcentaje de población en ranchos y casillas.

-Porcentaje de población con tenencia de obra social o mutual.

-Porcentaje de población que cuenta con descarga a red (Cloacas)

-Porcentaje de población que cuenta con conexión a gas de red.

Una vez seleccionadas las variables que intervendrán en el estudio, se procede con la operacionalización de las mismas. En ese sentido, se transforman los datos originales en 'puntajes estándar' (z).

Publicado en formato digital: Prof. Dr. Javier Gómez. CALIDAD DE VIDA Y DIFERENCIACION RESIDENCIAL EN EL GRAN SANTA FE A COMIENZOS DEL SIGLO XXI. RESÚMENES DE TESIS. Revista Geográfica Digital. IGUNNE. Facultad de Humanidades. UNNE. Año 12. N 24. Julio - Diciembre 2015. ISSN 1668-5180 Resistencia, Chaco

En: http://hum.unne.edu.ar/revistas/geoweb/default.htm 
Revista Geográfica Digital. IGUNNE. Facultad de Humanidades. UNNE. Año 12. № 24. Julio - Diciembre 2015. ISSN 1668-5180 Resistencia, Chaco

Del resultado obtenido se seleccionaron los cuatro componentes con autovalor mayor a 1 y que explican un $81,920 \%$ de la varianza contenida en las variables de partida, lo cual supone una importante síntesis de la información. Sin embargo, no todos tienen la misma capacidad explicativa. El primero da cuenta de algo más del $56 \%$ de la varianza, lo que lo sitúa a una apreciable distancia del segundo componente, que reúne algo menos del $12 \%$. El tercero, explica aproximadamente un $9 \%$ de la varianza, y el cuarto, algo más del $5 \%$.

Un total de 10 variables aportan significado al Componente 1 , y refieren a diferentes aspectos de la población siendo la mayoría indicativas de una buena situación de instrucción de la población y una inclusión con importante calificación en el mercado laboral. Ello, junto a buenas condiciones de servicios y equipamientos públicos. En suma, lejos de expresar circunstancias económicas precarias, este factor da cuenta de áreas sociales que cuentan con cierta solvencia económica, junto con existencia de un ciclo familiar maduro y bien provistas de servicios de confort.

Queda evidenciada, la asociación de este componente con los factores 'estatus social' y 'estatus familiar', señalada para el caso de las ciudades del tercer mundo (Puyol et al, 1988). Se denominó a este primer componente como "estatus social elevado con ciclo familiar maduro y presencia de servicios".

La identificación de la segunda dimensión que subyace en la diferenciación socio residencial de la población del Gran Santa Fe es relativamente sencilla, desde el momento en que son ocho las variables que dan significado al Componente 2, de las cuales siete puntúan con signo positivo. Un primer grupo conformado por tres variables que cargan en positivo porcentaje de población en viviendas con piso de tierra, porcentaje de población que reside en casillas y ranchos y porcentaje de población en viviendas sin inodoro- sería indicativo de la existencia de carencias infraestructurales de las viviendas. Si a este grupo anexamos la puntuación negativa del indicador 'porcentaje de población que reside en casas A y departamentos', podríamos señalar que este componente indicaría la presencia de precariedad edilicia.

En forma conexa al grupo anterior, se ha obtenido la puntuación positiva en este Componente 2 del indicador 'porcentaje de población que reside en hogares hacinados', por lo que la precariedad edilicia se traduce también en déficit en las condiciones de habitabilidad. Ello contribuye a plantear que este Componente refiere a un grupo social que reside en viviendas estructuralmente precarias y deficitarias de servicios, y que a su vez, no tienen las dimensiones que sus ocupantes demandan. Podemos atribuir estos resultados a condiciones de infraestructura estructuralmente precarias de la población.

Dentro del Componente 2, se destaca un segundo grupo de variables. Por un lado, la puntuación positiva del 'porcentaje de población que ya no asiste a establecimiento educativo, con nivel de instrucción máximo alcanzado primario incompleto' es indicativo de la precariedad educativa de la población que ya no concurre a la escuela. Junto a este indicador, el 'porcentaje de hogares con ambos jefes de hogar desocupados' es indicativo de la precaria situación laboral de la población económicamente activa y por último, la puntuación positiva del 'porcentaje de población con NBI capacidad de subsistencia' expone, consecuentemente, la insolvente condición económica de los grupos sociales involucrados. En conjunto, todos estos últimos indicadores apuntan en la misma dirección es decir, carencias estructurales de instrucción, de ocupación y de solvencia material de la población, junto al déficit en las viviendas en que reside. Se denominó al Componente 2 como "pobreza estructural".

En cuanto a la tercera dimensión de la diferenciación, es posible señalar que tan sólo son dos las variables que obtienen sus mayores saturaciones. Son los casos del 'porcentaje de población que hace cinco años residía en otra provincia argentina' y el 'porcentaje de población nacida en otra provincia argentina'. En muy menor medida se denota la carga

Publicado en formato digital: Prof. Dr. Javier Gómez. CALIDAD DE VIDA Y DIFERENCIACION RESIDENCIAL EN EL GRAN SANTA FE A COMIENZOS DEL SIGLO XXI. RESÚMENES DE TESIS. Revista Geográfica Digital. IGUNNE. Facultad de Humanidades. UNNE. Año 12. NN 24. Julio - Diciembre 2015. ISSN 1668-5180 Resistencia, Chaco

En: http://hum.unne.edu.ar/revistas/geoweb/default.htm 
positiva de la población con estudios universitarios completos, población con tenencia de obra social y población con gas de red. El Componente 3, por tanto, refiere primordialmente a la existencia de grupos de población de inmigrantes, en este caso, provenientes del fuera de la provincia de Santa Fe, es decir, de origen 'nacional'. Las cargas positivas en el resto de las variables citadas, junto a la puntuación negativa (aunque baja) del indicador 'población que ya no asiste con estudios primarios incompletos', permiten señalar que tal grupo de inmigrantes contaría con relativo buena situación económica. Por ello, se identificó a este factor como "inmigración nacional".

Finalmente, con respecto a la última dimensión obtenida, el Componente 4, son dos las variables también que obtienen saturaciones elevadas, en todos los casos con signo positivo: el 'porcentaje de población que hace cinco años residía en otra localidad de la provincia de Santa Fe' y el 'porcentaje de población sin agua corriente'. En procura de lograr mayor definición de este Componente, tomamos como referencia otras variables, que aunque con menor puntuación, podrían aportar información. Son los casos de la puntuación positiva del 'porcentaje de población que reside en viviendas con piso de tierra' y de las cargas negativas del 'porcentaje de población con descarga a red' y de 'ambos jefes de hogar desocupados'. Este conjunto, señalaría primordialmente grupos de población inmigrantes provenientes del interior de la provincia de Santa Fe, con importantes carencias de servicios públicos y relativo déficit edilicio. Aunque las condiciones laborales, no serían extremadamente precarias. Sin embargo, en función de las variables de mayor puntuación en este Componente, condujo a denominarlo "inmigración provincial con carencia de servicios públicos básicos".

En primer lugar, se ha detectado un sector nuclear principal del AGSF caracterizado por ser el área de asentamiento del estatus social alto con ciclo familiar maduro, y rodeando al mismo, un área intermedia que sirve de buffer hasta llegar al área constituida por los pobres estructurales con problemas de vivienda.

En dichas áreas, se asientan los inmigrantes nacionales y los provinciales. Aunque con patrones diferenciados entre si. En efecto, los inmigrantes nacionales tienden a una localización más restringida al municipio de Santa Fe, y los inmigrantes provinciales, se localizan preferentemente en los municipios periféricos y se asocia su asentamiento a la existencia de carencias sanitarias.

A su vez, se registra la existencia de radios de tipo 3 embutidos en el área de estatus social alto del municipio de Santa Fe. En un análisis más fino, las citadas incrustaciones de inmigración nacional en la mancha de estatus social elevado parecen estar asociadas, en contrapartida, con sectores de clase media-baja o baja. En efecto los radios de tipo 3 de localización en las áreas centrales coincide con la localización de las principales estaciones de transporte del AGSF: Estación Terminal de Ómnibus, ex estación ferroviaria del FCNGB. Y también a zonas de trazas ferroviarias actualmente desafectadas de su tradicional uso.

En segundo lugar, se revela una estructura urbana similar a la anterior en Santo Tomé, la segunda ciudad del AGSF. Por tanto, puede argumentarse la existencia de una estructura urbana general de tipo binuclear en el AGSF. Esta organización se ha detectado también en otros el estudio de Natera Rivas (2010), para los aglomerados de Tucumán y en Santiago del Estero -con La Banda del Río Salí y La Banda, respectivamente-.

En la Figura 46, se representan los radios censales a partir del componente o factor de mayor 'carga' en ellos. La intención es obtener una cartografía de síntesis, representativa de la estructura urbana interna del AGSF. Fue asignado, a cada unidad espacial, el componente en el cual ha presentado el mayor valor.

Los resultados obtenidos a partir del análisis de la estructura urbana del aglomerado del Gran Santa Fe como unidad urbana global, brindan una serie de pautas que señalan que la

Publicado en formato digital: Prof. Dr. Javier Gómez. CALIDAD DE VIDA Y DIFERENCIACION RESIDENCIAL EN EL GRAN SANTA FE A COMIENZOS DEL SIGLO XXI. RESÚMENES DE TESIS. Revista Geográfica Digital. IGUNNE. Facultad de Humanidades. UNNE. Año 12. N 24. Julio - Diciembre 2015. ISSN 1668-5180 Resistencia, Chaco

En: http://hum.unne.edu.ar/revistas/geoweb/default.htm 
Revista Geográfica Digital. IGUNNE. Facultad de Humanidades. UNNE. Año 12. № 24. Julio

- Diciembre 2015. ISSN 1668-5180 Resistencia, Chaco

disposición estaría en línea con lo supuesto por las principales modelizaciones de ciudades latinoamericanas de Ford (1996), Mertins (1995) y Janoschka (2002).

\section{MODELIZACION DE LA ESTRUCTURA URBANA DEL AGSF}

Los resultados previos permiten establecer rasgos estructurantes de la configuración espacial de los diversos grupos socioeconómicos en el AGSF. La diferenciación social y residencial santafesina puede ser interpretada a partir de los rasgos de zonificación que han sido postulados en los modelos de estructura interna propuestos por Ford (1996), Mertins (1995) y Janoschka (2002). De hecho, la estructura santafesina 'combina' esos modelos. Sin embargo, presenta también sus particularidades. Tanto las cuatro 'categorías' de calidad de vida' como la implantación territorial de los factores de diferenciación socio-residencial expresan una zonificación anillada que, partiendo del 'centro' alcanza la 'periferia'. Esta sucesión de anillos contiguos presenta notable similitud con la disposición en 'anillos' de las modelizaciones urbanas latinoamericanas. En el corazón de la ciudad se emplaza el denominado $C B D$, es decir el centro comercial del aglomerado. A continuación, el primer anillo, que rodea al $C B D$, estaría habitado por los grupos sociales más favorecidos desde el punto de vista socioeconómico, contiene a la vez las zonas urbanas de mayor madurez, es decir las mejor equipadas y que cuentan con mayor disponibilidad de servicios. Ello quedó evidenciado por el área de 'más favorable' calidad de vida y por las puntuaciones 'altas' y 'muy altas' en el Factor 1. Puntualmente, los resultados del análisis factorial indican que el $C B D$ en el AGSF no presenta las más altas puntuaciones, sino que éstas se ubican a modo de primer anillo del mismo, denominado 'residencial de elite'.

Se brindaron evidencias acerca de la existencia de un segundo anillo, que rodea al anterior, habitado principalmente por los grupos sociales medios, sobre un área urbana de cierta consolidación tanto de las infraestructuras como de los servicios y equipamiento (evidenciada por la categoría 'favorable' de calidad de vida' y las puntuaciones medianoaltas en el factor I), la cual respondería a la denominada 'área de madurez'.

Ha sido posible señalar la existencia de un tercer anillo que engloba a los grupos sociales desfavorecidos en un medio urbano con relativa presencia de equipamiento y servicios, asimilable al área de calidad de vida 'desfavorable' y las puntuaciones medio-bajas en el factor I 'estatus social elevado con ciclo familiar maduro'. Tales indicios señalarían compatibilidad con el anillo de 'acrecentamiento in situ' postulado por Ford (1996). En algunos tramos se constituye de barrios que comenzaron a surgir hacia las décadas de 1950 y 1960 (en los casos más antiguos) y que se fueron ampliando en las décadas subsiguientes. Describe un paisaje urbano caracterizado por una importante ocupación del suelo, aunque con espacios vacantes, casas en proceso de edificación y tendidos de servicios públicos escasos, que en conjunto presenta una escasa consolidación urbanística. Amplios sectores urbanos de Santa Fe y de Santo Tomé son compatibles con los caracteres explicitados para este anillo.

La estructura anillada, finalizaría en el exterior con un anillo representativo en buena parte, de los grupos sociales más desfavorecidos en un medio urbano con casi nulos servicios y equipamiento, lo que coadyuva en dotarlo de un paisaje de precariedad. Se correspondería con el área de calidad de vida 'más desfavorable', las puntuaciones bajas y muy bajas en el factor I 'estatus social elevado con ciclo familiar maduro' y con las más altas puntuaciones en el factor II 'pobreza estructural'.

Estrictamente, este cuarto anillo es urbanísticamente heterogéneo. En algunos casos, estaría representado por barrios aparecidos hacia fines de la década de 1940, en otros, por barrios representativos de la expansión horizontal de las tres últimas décadas del AGSF.

Publicado en formato digital: Prof. Dr. Javier Gómez. CALIDAD DE VIDA Y DIFERENCIACION RESIDENCIAL EN EL GRAN SANTA FE A COMIENZOS DEL SIGLO XXI. RESÚMENES DE TESIS. Revista Geográfica Digital. IGUNNE. Facultad de Humanidades. UNNE. Año 12. NN 24. Julio - Diciembre 2015. ISSN 1668-5180 Resistencia, Chaco

En: http://hum.unne.edu.ar/revistas/geoweb/default.htm 
La estructura básica anillada se sucede únicamente en las localidades de Santa Fe y en Santo Tomé, hecho que da lugar a postular la existencia de una estructura binuclear en el AGSF.

La escasa definición de la estructura anillada en el resto de las localidades del AGSF se explica por la 'juventud' de esas zonas y su reciente ocupación, lo cual redunda en escasos procesos de diferenciación interna en la escala en la cual trabajamos.

A la estructura anillada previamente explicada, debe anexarse una segunda, caracterizada por una expansión 'sectorial'. Este rasgo, aparece con claridad en la ciudad de Santa Fe, y consiste en la conformación de la expansión sectorial del 'grupo social de élite' desde el $C B D$ hacia la periferia, a través de los ejes de Boulevard Gálvez y las Avenidas Siete Jefes y Almirante Brown. En efecto, este sector, irrumpe sobre la estructura básica anillada, trastocando su sucesión natural de gradiente negativo hacia la periferia. De este modo, el grupo de 'elite', que como habíamos señalado previamente se afinca conformando un anillo que rodea al $C B D$, de modo complementario presenta una expansión sectorial que alcanza la periferia, en el extremo noreste de la ciudad principal del AGSF. Este elemento, aparece en las modelizaciones de metrópolis latinoamericanas de Ford (1996) y Mertins (1995). Ambas propuestas plantean la existencia de un 'mall' o 'subcentros comerciales' en el remate del sector de 'élite', que no es observable en el caso de Santa Fe. Si en cambio, tales amenidades periféricas aparecen hacia el este de Santa Fe, en la dirección de las casas de fin de semana, sobre el eje de la R.N. $N^{0} 168$. En tanto, en las restantes localidades del AGSF no se observan indicios importantes de esta estructuración en 'sectores'.

Entre los elementos propios de la tercera estructuración, relacionados a los procesos de fragmentación (Janoschka, 2002; Borsdorf, 2003; Prèvot Schapira, 2000), se encontró el afincamiento de grupos sociales de 'élite' en la periferia, materializados por barrios impulsados por emprendimientos inmobiliarios y de características 'cerradas'. La presencia de estos grupos sociales, que podría ser entendida como una especie de 'enclave', sería indicativa del inicio de una conformación de una estructura 'celular' periférica en el AGSF, según lo señalado por Janoschka, protagonizada por grupos sociales de 'elite', en un entorno socialmente deprimido.

\section{CONCLUSIONES}

Este trabajo ha logrado bosquejar la estructura de la diferenciación socioeconómica del AGSF a la luz de los modelos propuestos para las ciudades latinoamericanas y, en ese sentido, es posible señalar que los resultados indican la existencia conjunta de elementos propios de los tres modelos relevantes de estructura urbana que fueron tomados como marco en esta investigación, lo cual abona la validez de la primera hipótesis planteada.

Con respecto a la segunda hipótesis, el análisis de ecología factorial permitió demostrar que detrás de la localización diferenciada de los grupos sociales se encuentra dos factores asociados al estatus social: el 'estatus social elevado con ciclo familiar maduro' y la 'pobreza estructural'. Mientras, los factores 3 y 4 , de raigambre migratoria -no racial, sino con tintes también socioeconómicos- serían parcialmente comparables al factor 'racial' clásico dado que no evidencia el origen étnico de la población, sino su procedencia, denotándose fundamentalmente el 'peso' migratorio.

Es conveniente señalar que es insoslayable asociar, en parte, la localización de los grupos sociales más desfavorecidos con las condiciones del sitio del AGSF. En efecto, buena parte de los sectores periféricos del ASGF se encuentran enclavados en terrenos de escasa altitud, como es el caso del 'cordón' oeste del municipio capitalino, situado sobre el valle de inundación del río Salado, actualmente urbanizado en condiciones de precariedad, y

Publicado en formato digital: Prof. Dr. Javier Gómez. CALIDAD DE VIDA Y DIFERENCIACION RESIDENCIAL EN EL GRAN SANTA FE A COMIENZOS DEL SIGLO XXI. RESÚMENES DE TESIS. Revista Geográfica Digital. IGUNNE. Facultad de Humanidades. UNNE. Año 12. NN 24. Julio - Diciembre 2015. ISSN 1668-5180 Resistencia, Chaco

En: http://hum.unne.edu.ar/revistas/geoweb/default.htm 
defendido de las crecidas mediante terraplenes, que además de no ser infalibles, provocan un efecto embalse de las aguas generadas por precipitaciones, que ha conllevado la instalación de un complejo sistema de extracción mecánico, que en ocasiones también ha manifestado fallas.

Una situación similar muestra, en tanto, el conjunto de barrios localizados hacia el este del municipio capitalino, que junto a los distritos de San José del Rincón y Arroyo Leyes.

Se considera que este estudio se constituye como un antecedente válido para estudios futuros, que podrían abordar la temática no sólo desde un punto de vista cuantitativo, sino también avanzar desde las miradas más cualitativas. En ese sentido, nuevos estudios que adopten enfoques de base cualitativa, podrían profundizar aún más en el conocimiento de la diferenciación intraurbana del AGSF y lograr una aproximación mayor a los aspectos espaciales de las problemáticas sociales. Dado que esa tarea es un desafío, este estudio podría, y así lo pretende, contribuir a servir de base para trazar nuevas líneas de investigación en tal sentido.

Al respecto, dado que el aglomerado presenta una organización compleja y con notables particularidades, se estima que mediante nuevas indagaciones que adopten un área de estudio más acotada, o una escala más micro, podrían encontrarse nuevas evidencias acerca de la diferenciación intraurbana del AGSF o bien, profundizarse ciertos aspectos.

\section{BIBLIOGRAFÍA}

BÄHR, Jürgen y MERTINS, Günter (1982) A model of the social and spatial differentiation of Latin American metropolitan cities. Applied Geography and Development, 19. Tubingen: Institute for Scientific Cooperation.

BORSDORF, Axel (2003) Cómo modelar el desarrollo y la dinámica de la ciudad latinoamericana. Eure, v. 29, n. 86. Santiago de Chile.

BUZAI, Gustavo (2003a) Análisis y evaluación territorial de la situación socio-habitacional de la ciudad de Luján. Un estudio de ecología factorial urbana. Serie Avances de Investigación, año 1, n. 1. Luján: Universidad Nacional de Luján.

BUZAI, Gustavo (2003b) Mapas sociales urbanos. Buenos Aires: Editorial Lugar.

BUZAI, Gustavo (2004) Distribución, segregación y autocorrelación espacial de extranjeros en la ciudad de Luján (Argentina). Avances de Investigación, año 2, n. 4. Luján: Universidad Nacional de Luján.

BUZAI, Gustavo y BAXENDALE, Claudia (2006) Análisis socio-espacial con Sistemas de información Geográfica. Gepama. Buenos Aires: Editorial Lugar.

CERVERA, Felipe (1970) Ecología de la ciudad de Santa Fe. Santa Fe: Universidad Nacional del Litoral.

ESTEBANEZ, José (1991) Tendencias y problemática actual de la geografía, Serie Geografía, Cuadernos de Estudio № 1. Madrid: Cincel.

FERRER, Aldo (1973) La economía argentina ( $2^{\circ}$ edición actualizada del original de 1963). Buenos Aires: Fondo de Cultura Económica.

FERRER, Aldo y ROUGIER, Marcelo (2008) La economía argentina: desde sus orígenes hasta principios del siglo XXI. Buenos Aires: Fondo de Cultura Económica.

FORD, Larry (1996) A new and improved model of Latin American city structure. Geographical Review, n. 86 (3), 437-440. Nueva York: American Geographics Society.

GALLARDO, Mabel (1995) La Geografía y las periferias urbanas. Anales de la Academia Nacional de Geografía, n. 19, 211-221. Buenos Aires: Academia Nacional de Geografía. 
Revista Geográfica Digital. IGUNNE. Facultad de Humanidades. UNNE. Año 12. № 24. Julio - Diciembre 2015. ISSN 1668-5180 Resistencia, Chaco

GOMEZ, N., TARABELLA, L., PERETTI, G. y DEMARCHI, M. (2008) Composición de la población y calidad de vida en el municipio de Santa Fe. X Jornadas Cuyanas de Geografía. Mendoza: Universidad Nacional de Cuyo.

GRIFFIN, Ernst y FORD, Larry (1980) A model of Latin American city structure. Geographical Review. v. LXX, n. 4, 397-422. Nueva York: American Geographical Society.

GUTIERREZ PUEBLA, Javier (1992) La ciudad y la organización regional, Cuadernos de Estudio No 14. Serie Geografía (Reimpresión). Madrid: Cincel.

HAGGETT, Peter (1976) Análisis locacional en la geografía humana. Barcelona: Gustavo Gilli.

HARVEY, David (1992) Urbanismo y desigualdad social. Madrid: Siglo XXI.

HARVEY, David (2004) La condición de la posmodernidad, Investigación sobre los orígenes del cambio cultural. (1era. Reimp.) Buenos Aires: Amorrortu.

HOWELL, David (1989) A model of Argentine city structure. Revista Geográfica, v. 14, n. 109, 129140. México: I.P.G.H.

INDEC (1998) El concepto de localidad: definición, estudio de caso y fundamentos teóricos metodológicos. Buenos Aires: Autor.

INDEC (2001) Censo Nacional de Población, Hogares y Viviendas. Definición del concepto de 'Localidad'. Buenos Aires: Autor.

INDEC (2003) ¿Qué es el Gran Buenos Aires? Buenos Aires: Autor.

JANOSCHKA, Michael (2002) El nuevo modelo de la ciudad latinoamericana: fragmentación y privatización. Eure, v. 28, n. 85. Santiago de Chile.

KAZTMAN, Rubén (1999) Segregación residencial y desigualdades sociales en Montevideo. LC/MVD/R.177/Rev.1. Montevideo: CEPAL.

LOBATO, Mirta y SURIANO, Juan (2004) Nueva Historia Argentina. Buenos Aires: Sudamericana.

MERTINS, Günter (1995) La diferenciación socioespacial y funcional de las ciudades intermedias latinoamericanas: ejemplos del noroeste argentino. Revista Interamericana de Planificación, n. 112.

MIGNONE, Marcelo (2009) La segregación residencial socioeconómica en las capitales provinciales del nordeste argentino entre 1991 y 2001. X Jornadas Argentinas de Población AEPA. San Fernando del Valle de Catamarca.

MILANESIO DE REÑE, María L. (1994) Estructura interna de Rosario: Aplicación de un modelo. Contribuciones Científicas, 55 Semana de Geografía. Rosario: Sociedad Argentina de Estudios Geográficos. GAEA.

MUNICIPALIDAD DE LA CIUDAD DE SANTA FE. SECRETARÍA DE CULTURA. Municipalidad de Santa Fe (2008) Fascículo 5, Santa Fe, la ciudad y el río. Aula Ciudad. Santa Fe: Autor.

NATERA RIVAS, Juan J. (1998) Urbanización de la pobreza. Procesos migratorios y formación de periferias de invasión en una ciudad intermedia argentina. Málaga: Dpto. de Geografía de la Universidad de Málaga.

NATERA RIVAS, Juan J. (2002) Heterogeneidad social y diferenciación residencial en Marbella. Málaga: Biblioteca Popular Malagueña.

NATERA RIVAS, Juan J. (2005) Patrones de localización socio habitacional urbana en San Miguel de Tucumán. Breves Contribuciones del I.E.G., n. 17, 17-54. Tucumán: Instituto de Estudios Geográficos, UNT.

NATERA RIVAS, Juan J. (2010) Diferenciación socio residencial del espacio urbano en las capitales provinciales del Noroeste Argentino. Revista del Departamento de Geografía, n. 11, 45-65. Tucumán: Ed. Universidad Nacional de Tucumán.

NATERA RIVAS, Juan J. y GÓMEZ, Néstor J. (2007) Diferenciación Residencial en el Aglomerado Gran Santa Fe. Revista Universitaria de Geografía, v. 16, 99-124. Bahía Blanca: Universidad Nacional del Sur, Depto. de Geografía y Turismo.

Publicado en formato digital: Prof. Dr. Javier Gómez. CALIDAD DE VIDA Y DIFERENCIACION RESIDENCIAL EN EL GRAN SANTA FE A COMIENZOS DEL SIGLO XXI. RESÚMENES DE TESIS. Revista Geográfica Digital. IGUNNE. Facultad de Humanidades. UNNE. Año 12. NN 24. Julio - Diciembre 2015. ISSN 1668-5180 Resistencia, Chaco

En: http://hum.unne.edu.ar/revistas/geoweb/default.htm 
Revista Geográfica Digital. IGUNNE. Facultad de Humanidades. UNNE. Año 12. № 24. Julio

- Diciembre 2015. ISSN 1668-5180 Resistencia, Chaco

PRÉVÔT SCHAPIRA, Marie F. (2000) Segregación, fragmentación, secesión. Hacia una nueva geografía social de la aglomeración de Buenos Aires. Economía, Sociedad y Territorio, v. II, n. 7, 405431. Buenos Aires.

PROVINCIA DE SANTA FE. MINISTERIO DE GOBIERNO Y REFORMA DEL ESTADO. SECRETARÍA DE TECNOLOGÍAS PARA LA GESTIÓN (2008) Análisis del crecimiento poblacional. Santa Fe: Autor.

PUYOL, Rafael; ESTEBANEZ, José y MENDEZ, Ricardo (1988) Geografía Humana. Madrid: Cátedra. ROCCATAGLIATA, Juan A. (Coord.) (2008) Argentina. Una visión actual y prospectiva desde la dimensión territorial. Buenos Aires: Emecé.

RODRIGUEZ VIGNOLI, Jorge (2001) Segregación residencial socioeconómica: ¿Qué es?, ¿Cómo se mide?, ¿Qué esta pasando?, ¿importa? Serie Población y Desarrollo, n. 16. Santiago de Chile: CEPAL ECLAC.

RODRIGUEZ, Jorge; ARRIAGADA, Camilo (2004) Segregación residencial en la ciudad latinoamericana. Eure, v. XXIX, n 89. Santiago de Chile.

ROFMAN, Alejandro (2000) Desarrollo regional y exclusión social. Transformaciones y crisis en la Argentina contemporánea. Buenos Aires: Amorrortu Editores.

ROFMAN, Alejandro y ROMERO, Luis (1997) Sistema socioeconómico y estructura regional en la Argentina. Buenos Aires: Amorrortu Editores.

SABATINI, Francisco (2003) La segregación social del espacio en las ciudades de América Latina. Documentos del Instituto de Estudios Urbanos y Territoriales, Serie Azul, n. 35. Santiago de Chile: Pontificia Universidad Católica de Chile.

SABATINI, Francisco, CACERES, Gonzalo y CERDA, Jorge (2001) Segregación residencial en las principales ciudades chilenas: tendencias de las tres últimas décadas y posibles cursos de acción. Eure, v. 27, n. 82. Santiago de Chile: Pontificia Universidad Católica de Chile.

SANTOS, Milton (1990) Por una geografía nueva. Madrid: Espasa Calpe.

SANTOS, Milton (2000) Naturaleza del espacio, Técnicas y tiempo razón y emoción. Barcelona: Ariel. SAVRANSKY, Carlos, 2004, Pasión por la arquitectura: el camino hacia la empresa y el mercado en arquitectura y construcción. Buenos Aires: Nobuko.

SHARMA, Subhash (1996) Applied Multivariate Techniques. Canadá: Ed. John Wiley and Sons Inc.

SHEVKY, Eshref y BELL, Wendell (1974) Análisis de Area Social. En Theodorson, G, Estudios de Ecología Humana. T.1. Barcelona: Labor.

THEODORSON, A. G. (1974) Estudios de Ecología Humana. T. 1. Barcelona: Ed. Labor. Vol. 1.

TIMMS, Duncan (1976) El mosaico urbano. Hacia una teoría de la diferenciación residencial. Colección Nuevo Urbanismo. No 21. Ed. I.E.A.L.

TORCIDA, S.; MARINELLI, C.; CEPEDA, R. y WINZER, N. (1999) Una metodología tendiente a validar la hipótesis de contigüidad espacial en relación a la calidad de vida para ciudades intermedias. En V Jornadas de la Asociación de Estudios de Población Argentina. Luján: Universidad Nacional de Luján.

TORRADO, Susana (1994) Estructura social de la Argentina. 1945-1983 (2 ed.). Buenos Aires: Ediciones de la Flor.

TORRES, Horacio (1978) El mapa social de Buenos Aires en 1943, 1947 y 1960. Buenos Aires y los modelos urbanos. Desarrollo Económico, v. XVIII, n. 70, Versión electrónica publicada en www.educ.ar.

VALENTINUZZI DE PUSSETTO, Lilia (1996) El Barrio del Puerto, Colección Santa Fe, su gente, sus tiempos. Santa Fe: Ediciones Colmegna.

VAPÑARSKY, César (1995) Primacía y macrocefalia en la Argentina: la transformación del sistema de asentamiento humano desde 1950. Desarrollo Económico, v. 35, n. 138, 227-254. Buenos Aires.

VAPÑARSKY, César (1997) La división oficial del territorio argentino: ¿un rompecabezas insoluble? En Darío Cantón y Raúl Jorriat (Comp.) La investigación social hoy: a cuarenta años de la creación

Publicado en formato digital: Prof. Dr. Javier Gómez. CALIDAD DE VIDA Y DIFERENCIACION RESIDENCIAL EN EL GRAN SANTA FE A COMIENZOS DEL SIGLO XXI. RESÚMENES DE TESIS. Revista Geográfica Digital. IGUNNE. Facultad de Humanidades. UNNE. Año 12. NN 24. Julio - Diciembre 2015. ISSN 1668-5180 Resistencia, Chaco

En: http://hum.unne.edu.ar/revistas/geoweb/default.htm 
del Instituto de Sociología, UBA, (pp. 203-234). Buenos Aires: Universidad de Buenos Aires, Instituto de Investigaciones Gino Germani/Oficina de Publicaciones del CBC.

VAPÑARSKY, César y GOROJOVSKY, Néstor (1990) El crecimiento urbano en la Argentina. Buenos Aires: Grupo Editor Latinoamericano-IIED.

VELAZQUEZ, Guillermo (2001) Geografía, calidad de Vida y fragmentación en la Argentina de los noventa. Análisis regional y departamental utilizando SIG's. Tandil: CIG. UNICEN.

VELAZQUEZ, Guillermo (2008) Geografía y Bienestar. Buenos Aires: Paidós.

VELAZQUEZ, Guillermo y GARCÍA, María C. (1999) Calidad de Vida Urbana. Aportes para su estudio en Latinoamérica. Tandil: CIG. UNICEN.

VELAZQUEZ, Guillermo y GÓMEZ LENDE, Sebastián (2005) Desigualdad y calidad de vida en la Argentina (1991-2001). Aportes empíricos y metodológicos. Tandil: CIG. UNICEN.

VIGLIONE, Hebe (2003) Historia de la población de la provincia de Santa Fe, 1858-1970. Revista de la Junta Provincial de Estudios Históricos de Santa Fe, n. LXIII. Santa Fe.

VINUESA ANGULO, Julio y VIDAL DOMINGUEZ, María (1991) Los procesos de urbanización, Colección Espacios y Sociedades No 13. Madrid: Síntesis.

ZAPATA GOLLAN, Agustín (1971) La urbanización hispanoamericana en el Río de la Plata. Publicación n 6 del Departamento de Estudios Etnográficos y Coloniales. Santa Fe: Imprenta Oficial. ZARATE MARTIN, Antonio (1991) El espacio interior de la ciudad. Colección Espacios y Sociedades No 12. Madrid: Síntesis. 